\title{
Adding Remote Controller Functionality To Any Stereo
}

\author{
Joe Abraham \\ Department of Electronics andCommunication \\ Engineering \\ Jyothi Engineering College, Cheruthuruthy \\ Thrissur-679531, Kerala, India
}

\author{
Ambily Francis \\ Department of Electronics andCommunication \\ Engineering \\ Jyothi Engineering College, Cheruthuruthy \\ Thrissur-679531, Kerala, India
}

\begin{abstract}
Use of stereo has become common in our lives. They are used in cars, TVs, music players etc. And it is essential at least to control their volumes. Suppose there is a stereo amplifier which functions pretty well but it does not have a remote. It would be very annoying if its volume cannot be controlled. So this project is useful as it creates a device which makes use of any existing remote to control the volume. For controlling the volume, we use a volume controller IC. The electronic volume controller IC PT2258 is a digital potentiometer which can be controlled using I2C protocols. It is used to control the attenuation for every combination possible from 0 to $-79 \mathrm{~dB} / \mathrm{step}$. Universal IR receiver is used to decode the IR codes and the data will be transferred to the Arduino which in turn communicates with the IC PT2258 and controls the volume. The device also consists of two buttons, which are used to synchronize the IR code of the existing remote with the device. So the user will be able to use the device easily.
\end{abstract}

Keywords:

\section{INTRODUCTION}

The device designed is used to control the stereo which doesn't come up with a remote controller or a device which is so old that the remote controller is not available in the market. It will help the users to control the volume of any stereo with any remote they have got with them. So to be precise we can add up a remote controllable system to a normal computer speaker or any speaker that are readily available in the market which doesn't come with remote and we need to control the volume of the same using a remote controller.

The device makes use of an Arduino, IC PT2258 which is a digital potentiometer, Universal IR receiver, two switches and 4 audio jacks ( 2 for input and 2 for output). The Universal IR receiver receives the code and is given to the Arduino. Arduino takes the value and controls the IC PT2258.The volume is controlled by making attenuation in the channels. Thus the output of the device will be attenuated signal of the input.

The same device can also be made using a pulse detector and an analog potentiometer IC, but the problem is that, we need to provide a predefined remote controller along with the device. Else we have to redesign the pulse detector each time the user needs to use any different remotecontrollers. On using an Arduino, the predefined IR codes can be changed simply by pressing the switch in the device. By pressing the switch new IR values will be written to the device.

The main application of this device is that in a stereo, which does not come with any remote or whose remote is lost or damaged but they work fine manually, can be controlled with any available remote

\section{LITERATURE SURVEY}

\subsection{Humble Volume Control Circuit}

The humble volume control circuit can use to control the volume. Volume controls are usually implemented with a potentiometer configured as a voltage divider. The signal goes to lug 1, the output is connected to the wiper (lug 2) and the lug 3 is connected to ground. If we turn the pot shaft, more or less of the signal voltage is sent to either to ground (quieter) or to the output through the wiper (louder). This simple approach works well, but we are concerned with stereo, which has that pesky concept of two independent channels. But your standard pot only is a "single-gang" device: it only has one set of lugs.

There are many issues with dual gang pots. Potentiometers are not exact devices. Their tolerance ratings aren't terribly high, usually in the range of 10percentage-20percentage. That means that you can grab two $10 \mathrm{~K} \mathrm{ohm} \mathrm{pots} \mathrm{of} \mathrm{the} \mathrm{exact} \mathrm{same} \mathrm{part} \mathrm{and} \mathrm{manufacturer}$ and expect one pot to max out at $8 \mathrm{k}$ and the other at $12 \mathrm{k}$. This really isn $^{0} \mathrm{t}$ a big deal for most applications circuits are designed with part tolerances in mind.

Stereo input volume control with those variances. Each "gang" of the pot could be off by as much as 20 percentages, and matching of both gangs with a given shaft position could be way off also, Make sense in an empirical way. The result is that by using a cheap dual-gang pot as a volume control you are actually building in an unintentional balance control also. You can never really be sure if the left and right levels being reproduced are what they were when recorded. The problem is these variances can become quite pronounced when you are dealing a stereo volume control. But in our project attenuation is properly controlled by IC PT228 by different attenuation steps so that balance control problem is minor 


\subsection{Balanced Volume Control Circuit}

The volume balance control circuit is a circuit that using to control the music between its source and speakers. The circuit is based on LM1036N which is a DC controlled tone (bass/treble), volume and balance circuit for stereo applications in car radio, TV and audio systems. The main disadvantages are complexity in design and are expensive.

\subsection{Digital Volume Control Circuit}

Digital volume control circuit is the another popular circuit using the IC MAX5486.

MAX5486 is a 40K dual digital volume / balance controller that has a pushbutton interface. The IC has a built in bias voltage source that eliminated the need of an external circuitry for the same purpose and thereby by reduces external parts count. The IC also has an LED status indicator driver circuit which can be used for driving the status indicator LEDs which indicates the volume level and balance level. The IC can be operated from a single or dual power supply and is available in 24 pin TSSOP package. The volume control circuit based on MAX5486 can be applied in a lot application like personal audio systems, hand held audio devices, home theatre systems, car audio systems,computer audio systems etc. The IC is not really purposeful an accuracy will be less But the main advantages of our project is, we can control the volume, without the circuit in hand, using a remote. The synchronized working of both IR sensors controls volume with any remote. It is an easy and advanced method comparing with other old methods.

\section{CIRCUIT DIAGRAM}

\subsection{OPERATON}

There are two switches in the device. By pressing the first switch and by pressing any key in the remote controller will make IR code emitted by remote save as Volume up command. And by pressing the second switch and then pressing any switch in remote will make the IR code save as volume down command. Once the value is saved the device will be comparing these values with the received IR values. If an IR code is received is same as that of a volume up command, then device will decrease the attenuation. And if the IR code received is same as that of volume down command then the device will increase the attenuation. The universal IR receiver receives the IR code and is given to the Arduino. The Arduino compares the received values to the predetermined values. The Arduino accepts the IR code if it is a predetermined one else it ignores the IR code and receives the next IR code. If the IR code is predetermined one, then the Arduino compares it with volume up command and volume down command. An attenuation factor is defined in the program. If a volume up command is received, then the attenuation factor will be decreased and if a volume down command is received then the attenuation factor will be increased. The value of attenuation factor is limited between
0 to $79 \mathrm{db}$. Once attenuation factor has been changed it will be communicated to the IC PT2258 using SDA and SCL which are used for $\mathrm{I} 2 \mathrm{C}$ communication. For this wire library is used in Arduino. Attenuation factor is changed means that an attenuation is given in the output signal when compared to the input. This simply means the volume has been changed or decreased according to the attenuation factor. This is how the device controls.

\section{IC PT2258}

PT2258 is a 6 channel electric volume controller IC utilizing CMOS technology, specially designed for the new generation of AV multi-channel Audio system. The attenuation ranges 0 to $79 \mathrm{~dB}$ at $1 \mathrm{~dB}$ step, low noise high channel separation.

\section{BUS INTERFACE}

Data are transmitted to and from the microprocessor to the PT2258 via the SDL and SCL make up the bus interface. It should be noted that the pull up resistors must be connected to the positive supply voltage.

\section{DATA VALIDITY}

The Data on the SDA line is consider valid and stable only when the SCL signal is in high state the high and low state SDA line can only change when SCL signal is low.

Fig 2 : Internal Block Diagram of PT2258

\section{START CONDITIONS}

A Start Condition is activated when,

The SCL is set to HIGH and SDA shifts from HIGH to LOW State.

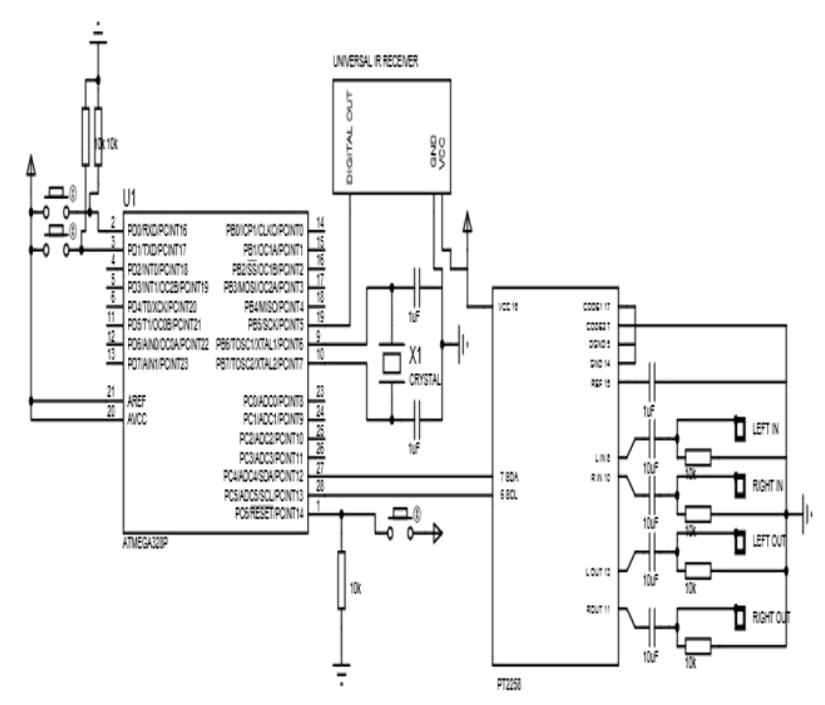

Figure 1: Diagram Representing the Whole of the Circuit Diagram 


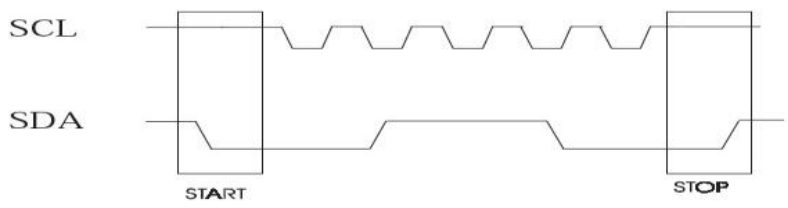

Figure 3: Start and Stop Condition Diagram

\section{STOP CONDITION}

The Stop Condition is activated when,SCL is set to HIGH and SDA shifts from LOW to HIGH State.Every byte transmitted to the SDA Line consists of 8 bits.

\section{SOFTWARE DESCRIPTION}

PT2258 Address Code depends on the state of CODE1 (Pin No. 17) and CODE2 (Pin No.4). If CODE1 or CODE2 is connected to Vcc, then CODE1 or CODE2 is set

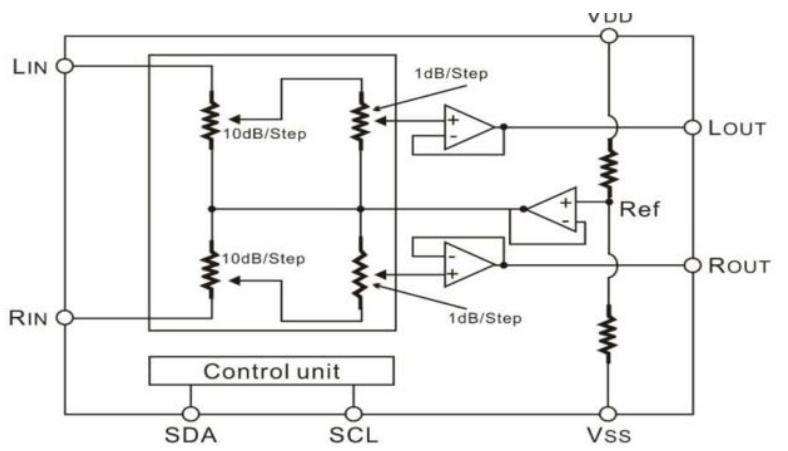

to 1. If CODE1 or CODE2 is connected to the Ground, it is set to 0. Data byte Description.Every byte transmitted to SDA line consist of 8 bites. Each byte must be followed by acknowledge bit. The MSB is transmitted first.

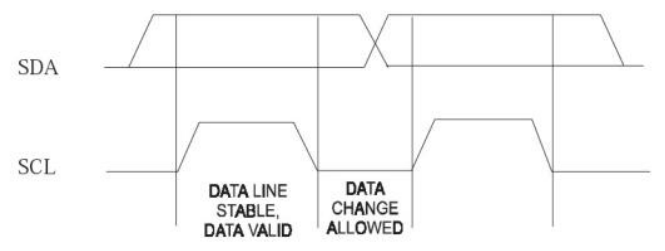

Figure 3: Start and Stop Condition Diagram

\section{FUNCTIONAL DESCRIPTION}

Data are transmitted to and from the microprocessor to the PT2258 via the SDA and SCL.The SDA and SCL make up the BUS Interface.A data on the SDA Line is considered valid and stable only when the SCL Signal is in
HIGH State. The HIGH and LOW States of the SDA Line can only change when the SCL signal is LOW.

\section{INTERFACE PROTOCOL}

- A start Condition

- A chip Address Byte including PT2258 address.8th bit of the byte must be 0 . It must always acknowledge the end transmitted byte.

- Data sequence (N-bytes+Acknowledge)

\section{○ IR Sensor}

IR used in this device is a universal IR receiver. Universal IR receiver is used to read the infra-red code. It converts the infra-red code to digital signal a transmits it through the digital pin to the micro-controller or microprocessor.

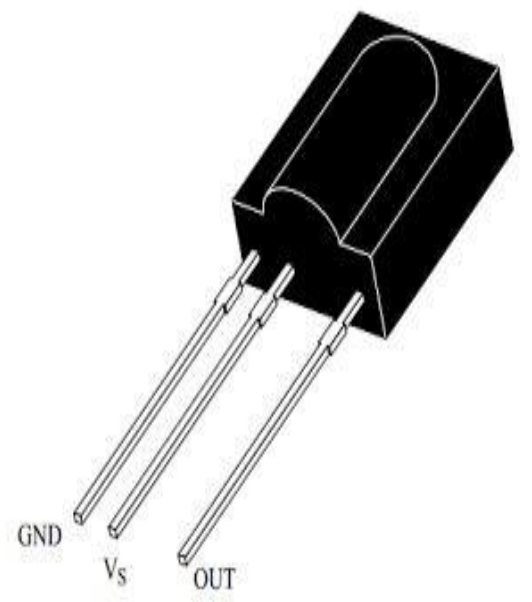

Figure 6: Pin Out of IR Sensor 


\begin{tabular}{|c|c|c|c|c|c|c|c|c|}
\hline $\begin{array}{l}M \\
\text { SB }\end{array}$ & & & & & & & $\begin{array}{l}\text { LS } \\
\text { B }\end{array}$ & \\
\hline 1 & 1 & 1 & 0 & A3 & $\mathrm{A} 2$ & $\begin{array}{l}A \\
1\end{array}$ & $\mathrm{~A} 0$ & $\begin{array}{l}\text { 6-Channel- - } \\
1 \mathrm{~dB} / \text { step }\end{array}$ \\
\hline 1 & 1 & 0 & 1 & 0 & B2 & $\begin{array}{l}B \\
1\end{array}$ & BO & $\begin{array}{l}\text { 6- channel- - } \\
\text { 10dB/step }\end{array}$ \\
\hline 0 & 0 & 0 & 1 & A3 & $\mathrm{A} 2$ & $\begin{array}{l}A \\
1\end{array}$ & $\mathrm{~A} 0$ & $\begin{array}{l}\text { channel No.3-- } \\
1 \mathrm{~dB} / \text { step }\end{array}$ \\
\hline 0 & 0 & 0 & 0 & 0 & B2 & $\begin{array}{l}B \\
1\end{array}$ & BO & $\begin{array}{l}\text { channel No.3- - } \\
10 \mathrm{~dB} / \text { step }\end{array}$ \\
\hline 0 & 0 & 1 & 1 & A3 & $\mathrm{A} 2$ & $\begin{array}{l}A \\
1\end{array}$ & $\mathrm{~A} 0$ & $\begin{array}{l}\text { channel no.4- - } \\
1 \mathrm{~dB} / \text { step }\end{array}$ \\
\hline 0 & 0 & 1 & 0 & 0 & B2 & $\begin{array}{l}B \\
1\end{array}$ & BO & $\begin{array}{l}\text { channel no.4- - } \\
10 \mathrm{~dB} / \mathrm{step}\end{array}$ \\
\hline 0 & 1 & 0 & 1 & A3 & $\mathrm{A} 2$ & $\begin{array}{l}A \\
1\end{array}$ & $\mathrm{AO}$ & $\begin{array}{l}\text { channel No.2-- } \\
1 \mathrm{~dB} / \text { step }\end{array}$ \\
\hline 0 & 1 & 0 & 0 & 0 & B2 & $\begin{array}{l}B \\
1\end{array}$ & BO & $\begin{array}{l}\text { channel No.2- - } \\
10 \mathrm{~dB} / \mathrm{step}\end{array}$ \\
\hline 0 & 1 & 1 & 1 & A3 & $\mathrm{A} 2$ & $\begin{array}{l}A \\
1\end{array}$ & $\mathrm{~A} 0$ & $\begin{array}{l}\text { channel No.5- - } \\
1 \mathrm{~dB} / \text { step }\end{array}$ \\
\hline 0 & 1 & 1 & 0 & 0 & B2 & $\begin{array}{l}B \\
1\end{array}$ & BO & $\begin{array}{l}\text { channel No.5- - } \\
10 \mathrm{~dB} / \text { decade }\end{array}$ \\
\hline 1 & 0 & 0 & 1 & A3 & $\mathrm{A} 2$ & $\begin{array}{l}A \\
1\end{array}$ & $\mathrm{~A} 0$ & $\begin{array}{l}\text { channel No.1- - } \\
1 \mathrm{~dB} / \text { step }\end{array}$ \\
\hline 1 & 0 & 0 & 0 & 0 & B2 & $\begin{array}{l}B \\
1\end{array}$ & BO & $\begin{array}{l}\text { channel No.1- - } \\
\text { 10dB/step }\end{array}$ \\
\hline 1 & 0 & 1 & 1 & A3 & $\mathrm{A} 2$ & $\begin{array}{l}A \\
1\end{array}$ & $\mathrm{~A} 0$ & $\begin{array}{l}\text { channel No.6- - } \\
1 \mathrm{~dB} / \text { step }\end{array}$ \\
\hline 1 & 0 & 1 & 0 & 0 & B2 & $\begin{array}{l}B \\
1\end{array}$ & BO & $\begin{array}{l}\text { channel No.6- - } \\
10 \mathrm{~dB} / \mathrm{step}\end{array}$ \\
\hline 1 & 1 & 1 & 1 & 1 & 0 & 0 & $M$ & $\begin{array}{l}\text { 6-Channel, } \\
\text { M=1 MUTE ON, } \\
\text { M=0 MUTE OFF }\end{array}$ \\
\hline
\end{tabular}

\section{ATTENUATION UNIT BIT}

\begin{tabular}{|c|c|c|c|c|}
\hline A3/... & A2/B2 & A1/B1 & A0/B0 & $\begin{array}{c}\text { ATTENUATION } \\
\text { VALUE(dB)+ }\end{array}$ \\
\hline 0 & 0 & 0 & 0 & $0 / 0$ \\
\hline 0 & 0 & 0 & 1 & $-1 /-10$ \\
\hline 0 & 0 & 1 & 0 & $-2 /-20$ \\
\hline 0 & 0 & 1 & 1 & $-3 /-30$ \\
\hline 0 & 1 & 0 & 0 & $-4 /-40$ \\
\hline 0 & 1 & 0 & 1 & $-5 /-50$ \\
\hline 0 & 1 & 1 & 0 & $-6 /-60$ \\
\hline 0 & 1 & 1 & 1 & $-7 /-70$ \\
\hline 1 & 0 & 0 & 0 & $-8 / \ldots$ \\
\hline 1 & 0 & 0 & 1 & $-9 / \ldots$ \\
\hline
\end{tabular}

\section{Arduino and Programming}

Algorithm

1. Start

2. Check the buttonstate1 and buttonstate2.If any one of them is high, save the IR code received as a and b respectively

3. Read the IR codes available at the IR sensor

4. Compare it with a and b.

5. If IR code received is a.

6. And if the attenuation factor is $i$ is greater than 0 , Then $\mathrm{i}=\mathrm{i}-5$, and jump to step 10

7. If IR code received is $b$

8. And if the attenuation factor is $i$ is lesser than 79 , Then $\mathrm{i}=\mathrm{i}+5$, and jump to step 10

9. If the IR code is not $a$ and $b$, then ignore them.

10. Split the attenuation factor $i$ to ones and tens.

11. Transmit this ones and tens to the PT2258 IC through I2C channels.

12. Jump to step2

\section{Hardware Description}

\section{IC PT2258}

PT2258 is a 6-Channel Electronic Volume Controller IC utilizing CMOS Technology specially designed for the new generation of AV Multi-Channel Audio System. PT2258 provides an $\mathrm{I} 2 \mathrm{C}$ Control Interface, an attenuation range of 0 to $-79 \mathrm{~dB}$ at $1 \mathrm{~dB} / \mathrm{step}$, low noise, high channel separation. Housed in 20-pin, DIP or SO Package, PT2258's pin assignments and' application circuit are optimized for easy PCB Layout and cost saving advantages.

The main features of IC PT2258 are as follows:

- CMOS Technology

- Low Power Consumption

- Least External Components

- Attenuation Range: 0 to $-79 \mathrm{~dB}$ at $1 \mathrm{~dB} / \mathrm{step}$

- Operating Voltage: 5 to $9 \mathrm{v}$ Low Noise, $\mathrm{S} / \mathrm{N}$ Ratio $>100 d B$ (A-weighting)

- High Channel Separation

- I2 C Bus Control Interface

- Selectable Address

- 6-Channel Outputs 


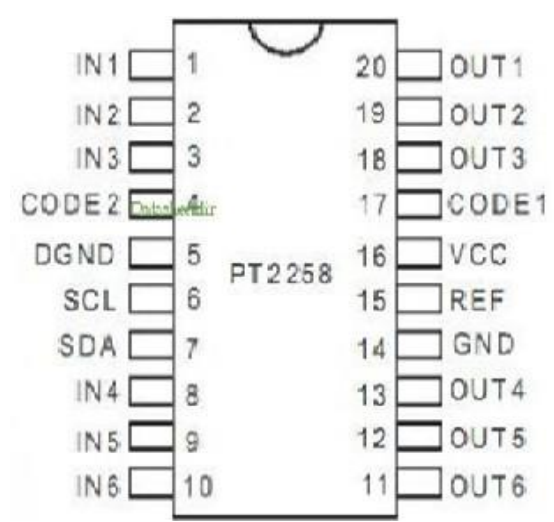

Figure 5.1: Pinout Diagram Of IC PT2258

IC PT2258 has any applications such as:

- AV Surround Audio Equipment

- Car Audio

- Mini Components

- Computer Multi-Media Speaker

- Other Audio Equipments

\section{IR Sensor}

An Infra red (IR) sensor is used to detect obstacles in front of the robot or to differentiate between colors depending on the configuration of the sensor. The picture shown is a very simple black box model of the IR Sensor. The sensor emits IR light and gives a signal when it detects the reflected light.It is a universal IR receiver which receivers all the IR codes that comes to it and it consist of 3 pins.It is a universal IR receiver which receivers all the IR codes that comes to it and it consist of 3 pins. Vcc, GND and digital out. Digital output gives the IR code received digitally. This is a simple infra red receiver which receives all the IR codes.It doesnt come specific.It is a universal on. It receives the IR code and transmit the received data digitally through out the digital pin.

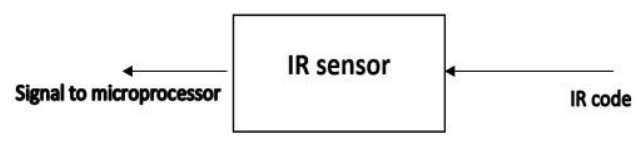

Figure 5.2: Ir Sensor Block Diagram

\section{RESULT}

The device was designed and implemented. And the device works fine with almost all remote controllers available in the market.

\section{CONCLUSION AND FUTURESCOPE}

A device is designed and implemented in such a way that it can use any existing remote, to control the volume of a remote less stereo or can be used instead of a damaged remote. In this project we have studied how to control the volume using volume control IC, PT2258. Basic understanding about the IR sensor and Arduino was also studied during this work. The design and verification of the remote control device was successful. The main advantage of this device is that any available remote can be used to control the volume of the required stereo just by pressing the buttons on the device implemented. Thus it is useful to operate even an old, good functioning stereo as it helps to control the volume. The project gave practical knowledge about the electronic circuit and components used in this work and helped to develop new ideas with proper guidance and help of facilities from the department. Project got completed successfully. Circuit can have further implementation in the future.

\section{REFERENCES}

[1] G. Eason, B. Noble, and I.N. Sneddon, "On certain integrals of Lipschitz-Hankel type involving products of Bessel functions," Phil. Trans. Roy. Soc. London, vol. A247, pp. 529-551, April 1955. (references)

[2] J. Clerk Maxwell, A Treatise on Electricity and Magnetism, 3rd ed., vol. 2. Oxford: Clarendon, 1892, pp.68-73.

[3] I.S. Jacobs and C.P. Bean, "Fine particles, thin films and exchange anisotropy," in Magnetism, vol. III, G.T. Rado and H. Suhl, Eds. New York: Academic, 1963, pp. 271-350.

[4] K. Elissa, "Title of paper if known," unpublished

[5] R. Nicole, "Title of paper with only first word capitalized," J. Name Stand. Abbrev., in press.

[6] Y. Yorozu, M. Hirano, K. Oka, and Y. Tagawa, "Electron spectroscopy studies on magneto-optical media and plastic substrate interface," IEEE Transl. J. Magn. Japan, vol. 2, pp. 740-741, August 1987 [Digests 9th Annual Conf. Magnetics Japan, p. 301, 1982]. 